\title{
CROSS-DOMAIN SEMI-SUPERVISED AUDIO EVENT CLASSIFICATION USING CONTRASTIVE REGULARIZATION
}

\author{
Donmoon Lee $e^{1,2}$, Kyogu Lee $e^{1,3}$ \\ ${ }^{1}$ Music and Research Group, Department of Intelligence and Information, \\ Seoul National University, \\ ${ }^{2}$ Cochlear.ai, ${ }^{3}$ Artificial Intelligence Institute, Seoul National University \\ \{lunideal,kglee\}@snu.ac.kr
}

\begin{abstract}
In this study, we proposed a novel semi-supervised training method that uses unlabeled data with a class distribution that is completely different from the target data or data without a target label. To this end, we introduce a contrastive regularization that is designed to be target task-oriented and trained simultaneously. In addition, we propose an audio mixing based simple augmentation strategy that performed in batch samples. Experimental results validate that the proposed method successfully contributed to the performance improvement, and particularly showed that it has advantages in stable training and generalization.
\end{abstract}

Index Terms - Audio event classification, semi-supervised learning, contrastive learning

\section{INTRODUCTION}

Sound contains a lot of information, but unfortunately, most of the audio-based studies have focused on speech. One of the barriers is the lack of a high-quality dataset. Since labeling audio is timeconsuming and expensive, it is difficult to establish a large-scale labeled audio dataset. Datasets such as AudioSet [1] acquire a large amount of data, but the quality of the labels is not guaranteed, which causes an additional problem, weakly supervised learning.

Self-supervised learning is one approach to use datasets with such large scale and unreliable labels. This can be done in a labelfree manner and the training is based on the assumption that the meaning of the data must remain in the presence of noise or transformations. It aims to learn general-purpose expressions that can be used anywhere without a target task and can be used for various downstream tasks through transfer learning or fine-tuning. Especially, the recent contrastive learning-based approach is promising as it shows similar or superior performance to supervised training in the image domain [2, 3]. In the case of the audio domain, various self-learning methods for speech have been proposed [4, 5], and recently, it has been expanded to various attempts targeting general audio [6, 7]. However, the downside is that self-supervised learning itself is so general that the performance of certain downstream tasks is not always guaranteed [8].

Semi-supervised learning [9] also utilizes unlabeled data. It differs from self-supervised learning in that there is a clear target task in the training procedure. In other words, the goal of semisupervised learning is to perform the target task with the help of unlabeled data. In general, most studies consider unlabeled data sets to contain a large number of target classes [10, 11]. Therefore, the focus is on finding available data in unlabeled datasets. In the

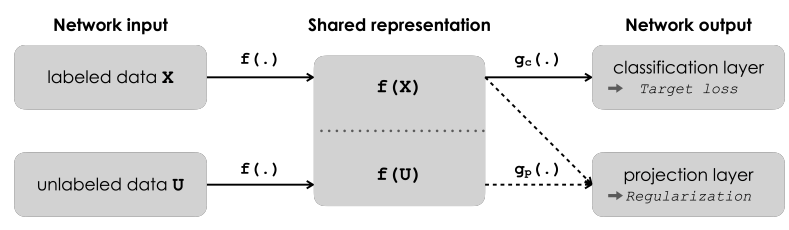

Figure 1: The overview of the proposed cross-domain semisupervised training. $f, g_{c}, g_{p}$ are the shared feature extractor, classification layer, and $\mathrm{L}^{2}$-normalized projection layer, respectively. The contrastive regularization is applied as an additional loss function.

implementation, the performance validation is done using labels for parts of large datasets. However, this assumption may not always be correct. Unlabeled data is literally unknown data, so there is no guarantee that the target data will be included.

In this study, we propose a cross-domain semi-supervised learning method that can be used when unlabeled data have a different class distribution than labeled data. The proposed method combines the concepts of semi-supervised and self-supervised training. More specifically, the network is trained to perform target tasks while regularized by unlabeled data using a contrastive learning approach. One advantage of our approach is that any unlabeled data can be used. It is also useful as it is applied in the form of additional losses, so it can be applied to almost all common networks. To the best of our knowledge, this is the first study to perform neural networkbased semi-supervised training using unlabeled data with different label distributions.

Our contributions are as follows:

- We propose a cross-domain semi-supervised training that can also be applied to data of completely different classes from the target data.

- We present a simple but efficient mixing strategy for applying contrastive learning to the audio domain, which is batch-split mixing.

\section{CONTRASTIVE REGULARIZATION FOR SEMI-SUPERVISED LEARNING}

The proposed semi-supervised method uses unlabeled data to learn differences between samples. As shown in Fig. 1 proposed regularization is performed in the form of multitask learning. However, it is different from the original multitask learning setup in that the regularization task uses a different unlabeled dataset than the target 


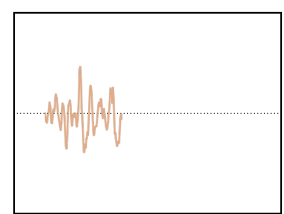

Original audio

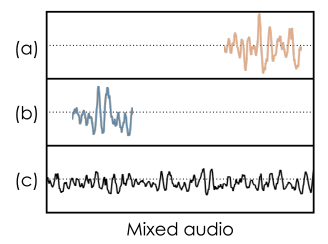

Figure 2: The proposed audio augmentation strategy. The original audio sample is augmented with a) random offset, b) mixing with another source, and c) adding artificial noise.

dataset. Rather, it is similar to the constraint method using other pre-trained networks [12], but the networks used for constraints are also trained at the same time. Such simultaneous training is expected to prevent common transfer learning problems such as catastrophic forgetting.

\subsection{Contrastive regularization}

Contrastive learning refers to a training method that recognizes identities and differences between each sample rather than classification through class labels [13, 14, 15]. This can also be applied to unlabeled data and is used to allow the network to learn semantics that is robust to data transformations. In particular, introducing NTXent loss [2] to solve contrastive learning as an in-batch classification is a promising method in recent self-supervised studies.

$$
N T \text { Xent }=-\log \frac{\exp \left(x \cdot x^{+} / \tau\right)}{\exp \left(x \cdot x^{+} / \tau\right)+\sum_{x^{-}} \exp \left(x \cdot x^{-} / \tau\right)}
$$

The proposed method introduces NTXent loss in semisupervised learning. It differs from the original method [2] in that the input of the NTXent loss is one original sample and one augmented sample. We postulate that it will be more useful to the target task as it directly includes the target dataset as opposed to the original method of assuming a more general-purpose task. This is a similar approach to the case where contrastive learning is used for a specific purpose. In [16], contrastive learning is applied to extract audio fingerprint. The learning process of contrastive learning can be viewed as a fingerprint task performed on a subset of the database. Therefore, in this case, the successful learning of contrastive learning guarantees the performance of the target task.

\subsection{Audio augmentation strategy}

We only used audio mixing as an augmentation method based on the unique properties of audio. One attribute of audio that differs from an image is that individual objects could be recognized even if multiple sources occur at the same time. Unlike an image where one masks the other when another source is added, the audio can be distinguished even if the two sources are mixed. We thought that this property alone could make audio augmentation difficult enough and could be applied to any kind of audio data. Audio mixing is often used in contrastive learning [6, 7], but mainly to add noise or background. The same idea as ours have been applied to tripletbased learning [17], but not to contrast learning, to the best of our knowledge.

To do this in a batch, we split the batch in two and mix it with another split. The mixing process involves mixing with random offsets, random amplitudes, and additional artificial noise, as shown

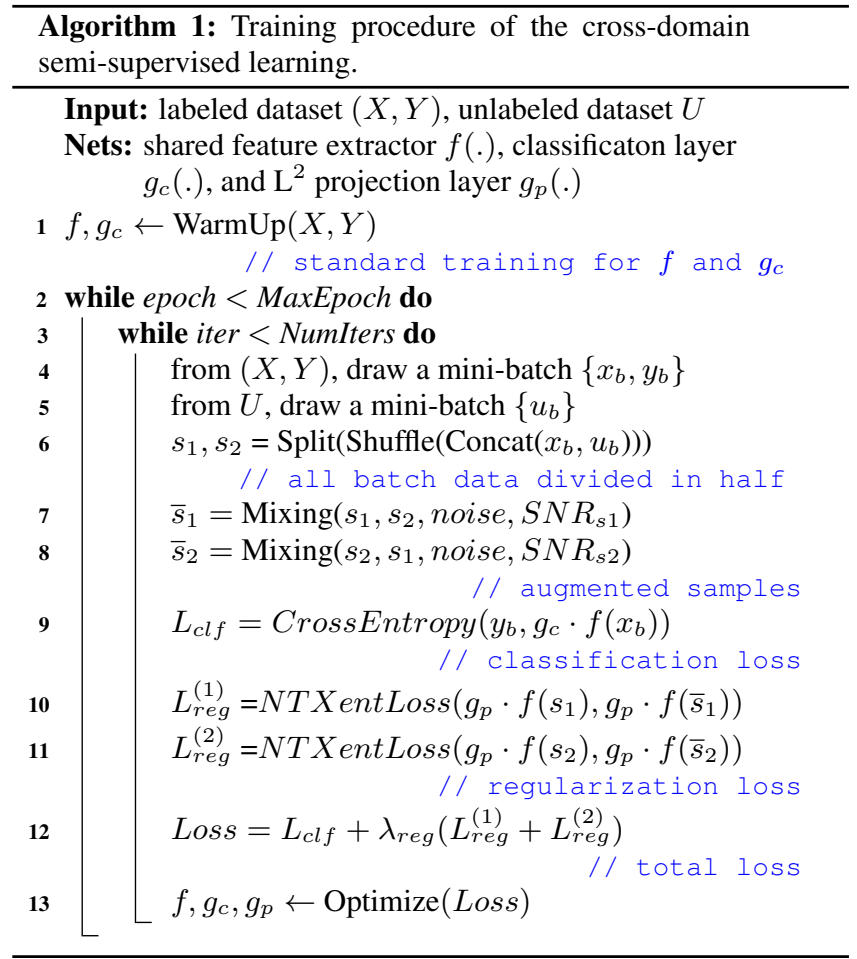

in Fig. 2. Audio events can be shorter than the length of the network input, so after the audio mixing process, they can exist individually on the time axis rather than as a mixture at the same time. This can be a relatively easy problem that only requires ensuring timedomain invariance, so additional noise is added to make it difficult. The reason for using artificial noise is that the traditional approach of using environmental sounds as noise, rather than the traditional target class [18], is limited in use by what the target sound is.

\subsection{Training procedure}

The actual training procedure is listed in Algorithm 1. At the first stage, the target classification network, which includes a shared feature extractor and classification layer, is initialized through warmup training. We found that training the network from scratch eventually yields similar performance, but takes more time to converge. Therefore, warm-up training is performed with a relatively high learning rate to shorten the training time.

In the semi-supervised learning stage, the labeled data is used for typical classification as well as warm-up training. The difference is that regularization that uses both labeled and unlabeled at the same time has been added. This results in NTXent loss after batch split mixing, as mentioned earlier. The total loss is expressed as the sum of the classification loss and regularization loss.

\section{EXPERIMENTS}

\subsection{Dataset}

- ESC10 and ESC50: The dataset for environmental sound classification [20] has a total of 2,000 audio samples for various audio events. ESC10 and ESC50 have 40 excerpts of 5 seconds for each class. It has the predefined 5-fold validation split. 
Table 1: The results for semi-supervised training using the data with mismatched label on the ESC10. Performance refers to the averaged test accuracy $(\%)$ and standard deviation for predefined 5-folds. best means the highest performance in the entire training epochs and last means the averaged performance of the last 20 epochs. Note that $\dagger$ is evaluated on different dataset distributions.

\begin{tabular}{c|c|ccccc}
\hline \multicolumn{2}{c|}{ Data fraction } & $10 \%$ & $25 \%$ & $50 \%$ & $75 \%$ & $100 \%$ \\
\hline Supervised & best & $59.0 \pm 5.0$ & $67.2 \pm 2.2$ & $75.0 \pm 2.9$ & $76.2 \pm 3.7$ & $81.2 \pm 1.6$ \\
& last & $53.0 \pm 5.8$ & $60.2 \pm 2.4$ & $65.9 \pm 1.6$ & $66.8 \pm 5.0$ & $72.3 \pm 1.3$ \\
\hline Supervised & best & $54.0 \pm 4.8$ & $76.2 \pm 7.1$ & $86.5 \pm 1.5$ & $89.5 \pm 1.7$ & $93.0 \pm 3.6$ \\
(ImageNet init.) & last & $49.9 \pm 4.8$ & $73.0 \pm 7.7$ & $82.5 \pm 1.7$ & $84.9 \pm 1.9$ & $89.1 \pm 3.6$ \\
\hline Proposed & best & $65.2 \pm 1.7$ & $78.5 \pm 3.7$ & $90.0 \pm 1.4$ & $93.0 \pm 1.3$ & $95.2 \pm 4.0$ \\
& last & $61.8 \pm 0.4$ & $75.6 \pm 3.2$ & $85.4 \pm 1.9$ & $87.6 \pm 3.2$ & $92.4 \pm 2.5$ \\
\hline Supervised [19] & & $67.8 \pm 4.0$ & $82.5 \pm 5.7$ & $88.3 \pm 3.0$ & - & $91.7 \pm 2.0$ \\
Deep-Co-Training $\lceil$ [19] & & $75.7 \pm 5.3$ & $89.2 \pm 4.0$ & $91.7 \pm 5.1$ & - & - \\
\hline
\end{tabular}

- US8K: The urban sound 8K [21] contains 8,732 labeled sound excerpts less than 4 seconds of 10 environmental sounds. The audio length varies from 50 milliseconds to 4 seconds and it has a predefined 10 -fold validation split.

\subsection{Network Architecture}

A network takes 5 -seconds of audio as input. It then converted to $\mathrm{dB}$-scale log mel spectrogram, a common feature used in audio analysis. For the STFT, the nfft size was 1,024 and the hop size was 230 , and the frequency information from $50 \mathrm{~Hz}$ to $10,000 \mathrm{~Hz}$ was converted to a 128-bin mel frequency. Thereafter, the mono channel mel spectrogram is stacked three times on the channel axis with the same value, to use the pre-trained weights [22] 23], resulting in the shape of $(128,480,3)$.

ResNet50 [24], which is often used in self-supervised studies [7. 25] with comparable performance in audio event classification, and is known to be effective for similar temporal unit analysis [26], was used as a feature extractor to output a 2,048-dimensional representation. The classification task branch consists of a set of 128dimensional fully connected layer, batch normalization, and $\operatorname{ReLU}$ activation, followed by the final output layer. The contrastive regularization branch is similarly connected through 128-dimensional fully connected layer, batch normalization, and $\operatorname{ReLU}$ activation, and as an output, an L2-normalized 64-dimensional embedding vector. The final output of this branch means an embedding for each data, comparisons with another embedding can be done through a dot product, the larger it means the two data are more similar.

\subsection{Experiment configuration}

Two experiments were conducted with data in and out of the dataset.

- Semi-supervised training using the data with mismatched label: ESC10 was used as labeled data. We defined ESC40 as ESC50 excluding ESC10 and used as unlabeled data in the proposed method. As with self-supervised studies [2], all unlabeled labels were always used, and parts of labels were used.

Deep-Co-Training $\dagger$ [19] is a method of pseudo-labeling unlabeled data using two networks that are trained to provide different predictive values and complement each other. A portion of ESC1O is used as labeled and the rest is used as unlabeled.

- Cross-dataset semi-supervised training: Both ESC50 and US8K were used in the experiment, one for labeled data and the other for unlabeled data. That is, when classifying ESC50, US8K was used for contrastive regularization and vice versa.

To reduce the influence of other variables in the experiment, no changes such as augmentation were made to the data pipe in target classification. The networks are initialized with the weights learned from the ImageNet. This is because the kernel trained to analyze the image is also known effectively for the classification of environmental sounds [22 23].

\subsection{Implementation details}

All audio data is resampled to $22,500 \mathrm{~Hz}$ and the scale is normalized based on energy. When each audio is used for network input, the short audio is zero-padded for up to 5 seconds. Audio mixing was done in the SNR range of $[-5,20] \mathrm{dB}$. A total of 6 artificial noises including blue, brown, grey, pink, violet, and white were used and added to less than $6 \mathrm{~dB}$.

Warm-up was performed until the training accuracy was high enough, ESC10 and ESC50 performed 20 epochs, and US8K performed 10 epochs. Adam optimizer [27] with learning rate 1e-4 is used for warm-up training and with learning rate 1e-5 used for semi-supervised training. For the proposed method, we trained the network 100 epochs with 8 labeled and 32 unlabeled samples, and for supervised learning, 200 epochs were used because the test accuracy was relatively slow to converge.

Weight for regularization loss $\left(\lambda_{\text {reg }}\right)$ is set to 0.05 taking into account the scale of the loss value. The temperature of the regularization loss is set to 0.01 . We experimentally confirmed that training was successful in the range of $[0.001,0.1]$. All experiments were implemented using Tensorflow [28], and the network structure and weights of ResNet 50V2 included in the library were used.

\section{RESULTS AND DISCUSSION}

\subsection{Cross-domain semi-supervised learning on ESC10}

The results for semi-supervised training using the data with mismatched labels are listed in Table 1 best is the average of the highest accuracy observed within the entire epochs at each fold and can be seen as the maximum performance that can be achieved in this experimental setup. last is the average accuracy of the last 20 epochs and is generally expected to be achieved. Except for $10 \%$, an almost constant performance difference is observed between supervised learning using different initialization methods. Initializing 
Table 2: Experimental results for cross-dataset semi-supervised training. Performance refers to the average accuracy and standard deviation for predefined $\mathrm{k}$-fold validation.

\begin{tabular}{ccc}
\hline Target data & Unlabeld data & Accuracy (\%) \\
\hline ESC10 & - & $89.1 \pm 3.6$ \\
ESC10 & ESC40 & $92.4 \pm 2.5$ \\
ESC50 & - & $73.6 \pm 1.0$ \\
ESC50 & US8K & $83.5 \pm 3.0$ \\
US8K & - & $78.6 \pm 4.3$ \\
US8K & ESC50 & $79.7 \pm 4.3$ \\
\hline
\end{tabular}

the weights from the image domain had a huge impact on the performance improvement, reconfirming that the kernel trained in the image domain still helped the spectrogram domain. However, the reversed performance at $10 \%$ shows that these weighted initialization methods may harm the network when data is scarce. In this case, since the data is very small, 3 samples per class, it is thought that the network focuses on other elements learned from images rather than semantic elements, but more research is needed.

The proposed method shows the highest accuracy in all experimental settings. Since the proposed method does not affect the classification layer structurally, it can be seen that a better semantic representation is induced by adding contrastive regularization. Multitask learning can improve target performance depending on the composition of the task [29, 30], and in this case, it can be seen that it behaved as we intended. It also shows that even if each branch uses data from a different label distribution, or a completely different dataset (Table 2), the multitask learning assumptions still work. The performance improvement of $U S 8 K$ is less than that of ESC50. Unlike ESC50, US8K consists of audio clips shorter than 5 seconds, and we assume that energy-based mixing is expected to adversely affect.

The proposed method has relatively low performance and performance improvement compared to regular semi-supervised training [19] that uses the rest of labeled data as unlabeled data. Excluding the $10 \%$ condition, the standard semi-supervised training shows a higher performance improvement and converges on performance with the full data at about $50 \%$. However, at $100 \%$, the proposed method showed higher performance even though there are no various performance improvement techniques. This shows the difference between the standard method and the proposed method. The standard method always uses the same class of data under strong assumptions, so it can reach the desired performance faster with less data, but there is a limit to the performance gain.

\subsection{Generalization effect}

We assume that the main factors in improving performance are stable training and generalization. The difference between last and best in the proposed method is always smaller than that of supervised, and smaller or similar to that of the weight initialized network. It implies that contrastive regularization covers the target task as we assumed and guides the network in a direction that helps generalization even when the data is scarce. These trends also appear in Fig 3 The supervised training shows a typical learning curve. On the other hand, in the proposed method, the training accuracy increases rapidly, and both training and test accuracy remain constant. The rapid training seems to be due to contrastive regularization as well as warm-up training. In addition to fast training set

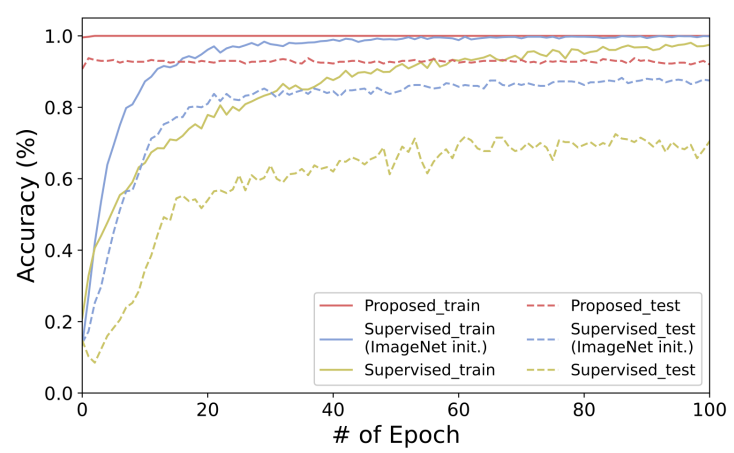

Figure 3: The learning curve for each learning method under the condition of using 100\% label data of ESC10. Each accuracy represents the average accuracy of each epoch in a 5-fold experiment.

convergence, preventing overfitting also seems to be an effect of the proposed method. High training accuracy was quickly achieved through warm-up training, but test accuracy was also consistently high through contrastive regularization. Through this, it is expected that much effort to find the optimal point could be reduced.

\subsection{Ablation study}

Table 3: Ablation study on ESC50 dataset.

\begin{tabular}{cccc}
\hline Proposed & -Mixing & -Unlabeled & Supervised \\
\hline $83.5 \pm 3.0$ & $82.0 \pm 3.0$ & $79.5 \pm 1.6$ & $73.6 \pm 1.0$ \\
\hline
\end{tabular}

Table 3 shows the results of excluding each element from the overall proposed method. In -Unlabeled condition, contrast regularization is added only within a labeled data set, with a performance improvement of 5.9\% points over the normal supervised condition. It shows that the proposed method is also helpful in a typical supervised setting. Without the data mixing strategy of the proposed method, -Mixing, which applies only time offset augmentation, there is a performance improvement of $8.4 \%$ points over the supervised condition. This implies that the increased diversity of tasks that external data can provide is a major factor in contrastive regularization.

\section{CONCLUSION}

In this study, we proposed cross-domain semi-supervised training that works with completely different data distributions. We introduced contrastive learning to the semi-supervised framework and proposed a novel augmentation method considering the audio characteristics. Experimental results have proven the effectiveness of the proposed method for audio event classification. Considering that the proposed method was implemented in a simplified form and the characteristics of the proposed method that it can be applied to any network, there is a lot of room for performance improvement. In particular, we expect that various augmentation methods and large-batch, which are the main factors in contrastive learning, may improve the performance further. 


\section{REFERENCES}

[1] J. F. Gemmeke et al., "Audio set: An ontology and humanlabeled dataset for audio events," in 2017 IEEE International Conference on Acoustics, Speech and Signal Processing (ICASSP). IEEE, 2017, pp. 776-780.

[2] T. Chen, S. Kornblith, M. Norouzi, and G. Hinton, "A simple framework for contrastive learning of visual representations," in International conference on machine learning. PMLR, 2020, pp. 1597-1607.

[3] T. Chen, S. Kornblith, K. Swersky, M. Norouzi, and G. Hinton, "Big self-supervised models are strong semi-supervised learners," arXiv preprint arXiv:2006.10029, 2020.

[4] S. Pascual, M. Ravanelli, J. Serrà, A. Bonafonte, and Y. Bengio, "Learning problem-agnostic speech representations from multiple self-supervised tasks," Proc. Interspeech 2019, pp. 161-165, 2019.

[5] S. Schneider, A. Baevski, R. Collobert, and M. Auli, "wav2vec: Unsupervised pre-training for speech recognition," Proc. Interspeech 2019, pp. 3465-3469, 2019.

[6] D. Niizumi, D. Takeuchi, Y. Ohishi, N. Harada, and K. Kashino, "Byol for audio: Self-supervised learning for general-purpose audio representation," arXiv preprint arXiv:2103.06695, 2021

[7] L. Wang and A. v. d. Oord, "Multi-format contrastive learning of audio representations," arXiv preprint arXiv:2103.06508, 2021.

[8] T. Xiao, X. Wang, A. A. Efros, and T. Darrell, "What should not be contrastive in contrastive learning," arXiv preprint arXiv:2008.05659, 2020

[9] X. Zhou and M. Belkin, "Semi-supervised learning," in Academic Press Library in Signal Processing. Elsevier, 2014, vol. 1, pp. 1239-1269.

[10] D. Berthelot, N. Carlini, I. Goodfellow, N. Papernot, A. Oliver, and C. Raffel, "Mixmatch: A holistic approach to semi-supervised learning," arXiv preprint arXiv:1905.02249, 2019.

[11] K. Sohn et al., "Fixmatch: Simplifying semi-supervised learning with consistency and confidence," arXiv preprint arXiv:2001.07685, 2020.

[12] J. Johnson, A. Alahi, and L. Fei-Fei, "Perceptual losses for real-time style transfer and super-resolution," in European conference on computer vision. Springer, 2016, pp. 694-711.

[13] J. Bromley, J. W. Bentz, L. Bottou, I. Guyon, Y. LeCun, C. Moore, E. Säckinger, and R. Shah, "Signature verification using a "siamese" time delay neural network," International Journal of Pattern Recognition and Artificial Intelligence, vol. 7, no. 04, pp. 669-688, 1993.

[14] R. Hadsell, S. Chopra, and Y. LeCun, "Dimensionality reduction by learning an invariant mapping," in 2006 IEEE Computer Society Conference on Computer Vision and Pattern Recognition (CVPR'06), vol. 2. IEEE, 2006, pp. 1735-1742.

[15] F. Schroff, D. Kalenichenko, and J. Philbin, "Facenet: A unified embedding for face recognition and clustering," in Proceedings of the IEEE conference on computer vision and pattern recognition, 2015, pp. 815-823.
[16] S. Chang, D. Lee, J. Park, H. Lim, K. Lee, K. Ko, and Y. Han, "Neural audio fingerprint for high-specific audio retrieval based on contrastive learning," in ICASSP 2021-2021 IEEE International Conference on Acoustics, Speech and Signal Processing (ICASSP). IEEE, 2021, pp. 3025-3029.

[17] A. Jansen, M. Plakal, R. Pandya, D. P. Ellis, S. Hershey, J. Liu, R. C. Moore, and R. A. Saurous, "Unsupervised learning of semantic audio representations," in 2018 IEEE international conference on acoustics, speech and signal processing (ICASSP). IEEE, 2018, pp. 126-130.

[18] J. Thiemann, N. Ito, and E. Vincent, "The diverse environments multi-channel acoustic noise database (demand): A database of multichannel environmental noise recordings," in Proceedings of Meetings on Acoustics ICA2013, vol. 19, no. 1. Acoustical Society of America, 2013, p. 035081.

[19] L. Cances and T. Pellegrini, "Comparison of deep co-training and mean-teacher approaches for semi-supervised audio tagging," in IEEE 46th International Conference on Acoustics, Speech and Signal Processing (ICASSP 2021), 2021.

[20] K. J. Piczak, "ESC: Dataset for Environmental Sound Classification," in Proceedings of the 23rd Annual ACM Conference on Multimedia. ACM Press, 2015, pp. 10151018. [Online]. Available: http://dl.acm.org/citation.cfm? doid $=2733373.2806390$

[21] J. Salamon, C. Jacoby, and J. P. Bello, "A dataset and taxonomy for urban sound research," in 22nd ACM International Conference on Multimedia (ACM-MM'14), Orlando, FL, USA, Nov. 2014, pp. 1041-1044.

[22] K. Palanisamy, D. Singhania, and A. Yao, "Rethinking cnn models for audio classification," arXiv preprint arXiv:2007.11154, 2020.

[23] A. Guzhov, F. Raue, J. Hees, and A. Dengel, "Esresnet: Environmental sound classification based on visual domain models," arXiv preprint arXiv:2004.07301, 2020.

[24] K. He, X. Zhang, S. Ren, and J. Sun, "Identity mappings in deep residual networks," in European conference on computer vision. Springer, 2016, pp. 630-645.

[25] J.-B. Grill, F. Strub, F. Altché, C. Tallec, P. H. Richemond, E. Buchatskaya, C. Doersch, B. A. Pires, Z. D. Guo, M. G. Azar, et al., "Bootstrap your own latent: A new approach to self-supervised learning," arXiv preprint arXiv:2006.07733, 2020.

[26] M. Won, A. Ferraro, D. Bogdanov, and X. Serra, "Evaluation of cnn-based automatic music tagging models," arXiv preprint arXiv:2006.00751, 2020.

[27] D. P. Kingma and J. Ba, "Adam: A method for stochastic optimization," arXiv preprint arXiv:1412.6980, 2014.

[28] M. Abadi et al., "TensorFlow: Large-scale machine learning on heterogeneous systems," 2015, software available from tensorflow.org. [Online]. Available: https://www.tensorflow. org/

[29] R. Caruana, "Multitask learning," Machine learning, vol. 28, no. 1, pp. 41-75, 1997.

[30] D. Lee, J. Lee, J. Park, and K. Lee, "Enhancing music features by knowledge transfer from user-item log data," in ICASSP 2019-2019 IEEE International Conference on Acoustics, Speech and Signal Processing (ICASSP). IEEE, 2019, pp. 386-390. 